\title{
Daytime reduction of gastro-oesophageal reflux after healing of oesophagitis and its value as an indicator of favourable response to maintenance treatment
}

\author{
F Pace, O Sangaletti, G Bianchi Porro
}

\begin{abstract}
In order to investigate the response of gastrooesophageal reflux after medically induced healing of oesophagitis and its relation to the occurrence of relapse during prophylactic treatment, 20 patients with erosive/ulcerative oesophagitis underwent 24 hour oesophageal pH monitoring before and after healing achieved with 12 to 24 week treatment with ranitidine 150 or $300 \mathrm{mg}$ twice daily. Compared with pretreatment values, after macroscopic healing, a significant reduction in daytime median percentage of reflux time $(10.0 v 6.5$ $p<0.05)$ and median number of reflux episodes lasting more than 5 minutes $(5-5 v 1.0 ; \mathrm{p}<0.05)$ were observed, whereas during night time reflux frequency and severity did not change. During maintenance treatment with ranitidine 150 or $300 \mathrm{mg}$ nocte, five of the six patients, who had shown no improvement in gastrooesophageal reflux after acute healing, relapsed. These results suggest that, in contrast with previous work, a decrease in gastrooesophageal reflux in patients with reflux oesophagitis can be achieved after macroscopic healing, and that the occurrence of such a reduction after acute healing is predictive of a good response to longterm treatment.
\end{abstract}

Endoscopic assessment of healing in patients with reflux oesophagitis seems to be simple and reliable, and therefore it is commonly used in clinical trials because macroscopic disappearance of mucosal lesions is considered to be an important objective of medical treatment. ${ }^{1}$ It is now clear, however, that mucosal repair and symptomatic relief do not necessarily behave in tandem with parallellism. ${ }^{2}$ It has also been observed that medically induced healing of reflux oesophagitis is not accompanied by the return to normal of histological features or by an improvement in motor function, as assessed by various tests. ${ }^{2-5}$ Lower oesophageal sphincter pressure, for instance, has been shown not to increase after successful treatment with $\mathrm{H}_{2}$ receptor antagonists, ${ }^{6}$ whereas it recovers after surgical treatment ${ }^{7}$; similarly, oesophageal peristaltic activity, ${ }^{3-5}$ acid clearance, ${ }^{2}$ and the acid perfusion (Bernstein) test $^{27}$ seem not to be consistently affected by healing.

These findings have led to the hypothesis that the oesophageal dismotility, which is considered to be an integral part of gastro-oesophageal reflux disease, ${ }^{8}$ may not be reversible with medical treatment ${ }^{2}$ and may contribute to the frequent occurrence of relapse in medically treated patients after symptomatic and endoscopic heal- ing. Other workers have proposed prolonging treatment beyond the disappearance of mucosal lesions and symptomatic relief in 'an attempt to improve mucosal inflammation and possibly also esophageal function.'

In the evaluation of gastro-oesophageal reflux, prolonged oesophageal $\mathrm{pH}$ monitoring seems to be the most sensitive and specific test avaialble, ${ }^{9}$ particularly if it is carried out for 24 hours. ${ }^{10}$ In only two of the studies quoted above,${ }^{+7}$ however, was oesophageal $\mathrm{pH}$ monitoring used to investigate whether gastro-oesophageal reflux was actually reduced after healing: in both studies the oesophageal $\mathrm{pH}$ profiles proved not to be significantly modified, but in both there were only a few patients (eight and eleven), and in the Lieberman's study ${ }^{7}$ some patients in fact had minimal or no oesophagitis (mucosal erythema) at the time of admission.

We therefore undertook a study in a larger series of ambulatory outpatients with erosive/ ulcerative oesophagitis, corresponding to Savary and Miller ${ }^{11}$ grade 1 to 4 , to verify whether 12 to 24 weeks of successful treatment with 150 or 300 mg ranitidine twice daily can modify the pattern of gastro-oesophageal reflux compared to the pretreatment level. In addition, we prospectively investigated whether in individual patients any improvement in gastro-oesophageal reflux can predict a successful response during subsequent prophylactic treatment with ranitidine.

\section{Methods}

PATIENTS

Twenty patients with reflux oesophagitis which had healed after treatment with ranitidine were studied. All 20 patients received 12 and 24 weeks of treatment with ranitidine, at a dose of 150 or $300 \mathrm{mg}$ twice daily, respectively, in a controlled clinical trial, the results of which are presented elsewhere (submitted). Informed consent was

TABLE I Characteristics of patients $(n=20)$. (Data are expressed as medians)

\begin{tabular}{lc}
\hline Age (years) & 50 \\
Sex & $1+$ men, 7 women \\
Duration of heartburn (years) & $3 \cdot 5$ \\
Treatment during acute study: & \\
$\quad$ Ranitidine $150 \mathrm{mg}$ twice daily (n) & 15 \\
$\quad$ Ranitidine $300 \mathrm{mg}$ twice daily (n) & 5 \\
Symptom score (none/mild/moderate/severe): & \\
$\quad$ Before treatment & $0 / 4 / 10 / 6$ \\
$\quad$ After treatment & $15 / 3 / 0 / 2$ \\
Endoscopy grading & 16 \\
$\quad$ Grade I \& II & 4 \\
$\quad$ Grade III \& IV & \\
Oesophageal pH $<4$ (\% of $24 \mathrm{~h}):$ & $10 \cdot 3$ \\
$\quad$ Before treatment & $7 \cdot 8$ \\
After treatment & \\
\hline
\end{tabular}

Gastrointestinal Unit, L Sacco Hospital, Milan, 
obtained from all the patients before treatment began. All were submitted to upper gastrointestinal fibre endoscopy before and after treatment; all were subsequently treated prophylactically for six to 12 months with half the dose of ranitidine given previously. Control endoscopy was performed after six months and, if there was no relapse, after 12 months. The severity of oesophagitis was scored according to the Savary and Miller classification" (grade $1=$ non-confluent superficial erosions; grade $2=$ confluent erosions, but not covering the whole circumference; grade $3=$ circumferentially confluent erosions; grade $4=$ presence of chronic complications, such as ulcers, stricture, Barrett's metaplasia).

The presence of hiatal hernia was endoscopically defined. Before they entered the study the history and symptoms of the patients were assessed and recorded.

Severity of heartburn was defined as: mild burning pain that resolves spontaneously; moderate - burning pain that resolves with one dose of antacids; severe - burning pain that persists despite at least one dose of antacids. Symptoms were assessed again on the same day that control endoscopy was performed - that is, after 12 or 24 weeks of treatment and after six or 12 months of longterm treatment.

\section{pH MONITORING STUDIES}

Each patient underwent 24 hour oesophageal pH monitoring before starting treatment. The technique is described elsewhere. ${ }^{10}$ Briefly, after an overnight fast a combined glass electrode (Ingold M440, Ingold, Switzerland) was passed transnasally after light pharyngeal anaesthesia with $2 \%$ lidocaine, passed into the stomach, and then withdrawn and positioned $5 \mathrm{~cm}$ above the gastrooesophageal junction, identified pH metrically. The outer end of the probe was then connected to a portable recorder (Autronicord CM 18, Autronic, FRG) and the position of the intraluminal tip of the probe checked fluoroscopically. The subjects could then leave the unit and come back 24 hours later to have the probe removed. During the test patients were instructed to have an average Italian diet, consisting in three meals of about 2000 calories in total. Each patient had exactly the same meal during the basal and the 24 hour $\mathrm{pH}$ monitoring studies after healing. The following parameters were calculated for the whole 24 hour period, and separately for daytime (0700 to 2300$)$ and night time ( 2300 to 0700 ): percentage time with $\mathrm{pH}<4$; number of reflux (oesophageal $\mathrm{pH}<4$ ) episodes; number of reflux episodes lasting more than five minutes. The $\mathrm{pH}$-metry was repeated within seven days of endoscopic evidence of healing, and after at least three days after ranitidine was discontinued ('wash-out' period).

\section{DATA ANALYSIS}

PH-metry data were analysed by nonparametrical statistical techniques, such as the Wilcoxon-White test, according to the recommendations recently published by a panel of international experts. ${ }^{12}$

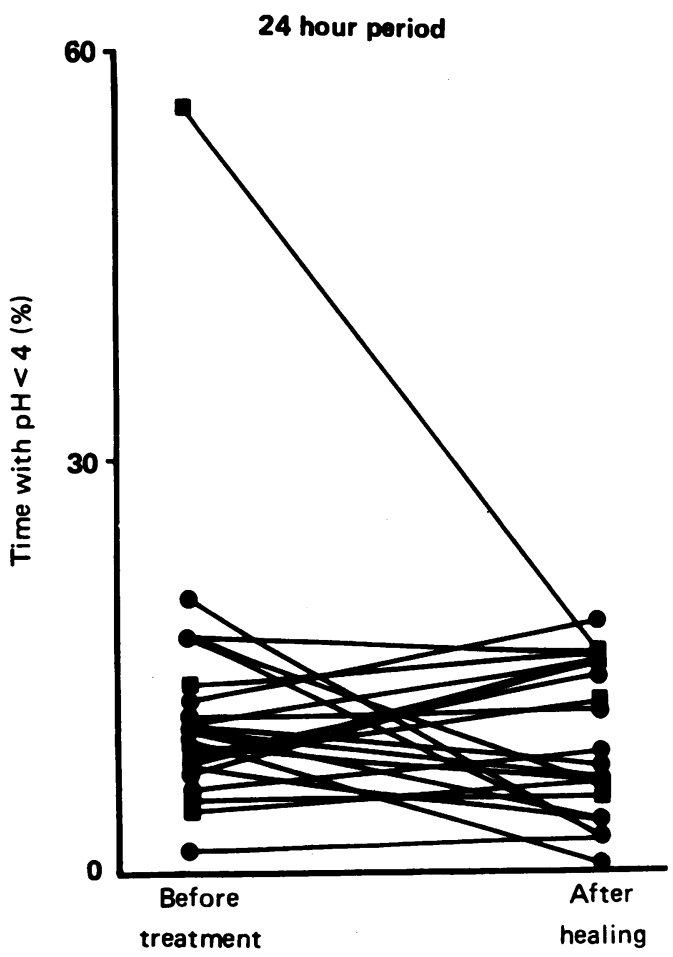

Figure 1: The percentage time with oesophageal $\mathrm{pH}<4$ during the total 24 hour period in the 20 patients with oesophagitis before treatment and after healing of the oesophageal mucosal lesions. Squares indicate a relapse during longterm treatment; circles indicate no relapse.

\section{Results}

Table I gives the patient characteristics. The median age was 50 years, with a duration of heartburn symptoms of 3.5 years. The oesophagitis was mainly mild, with only two patients with ulcerative oesophagitis. The pretreatment 24 hour $\mathrm{pH}$ recordings were noticeably abnormal in the population: the median percentage of time with a $\mathrm{pH}<4$ was 10.3 during the 24 hour test

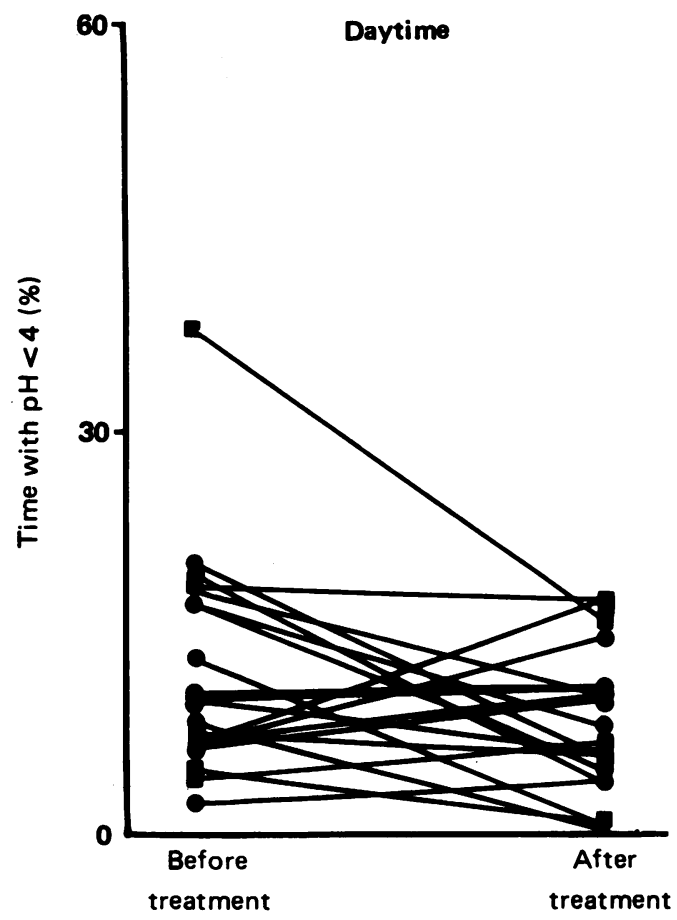

Figure 2: The percentage time with oesophageal $\mathrm{pH}<4$ during daytime $(16 h)$ in the 20 oesophagitis patients before treatment and after healing of oesophageal mucosal lesions. For explanations of symbols, see Fig 1. 


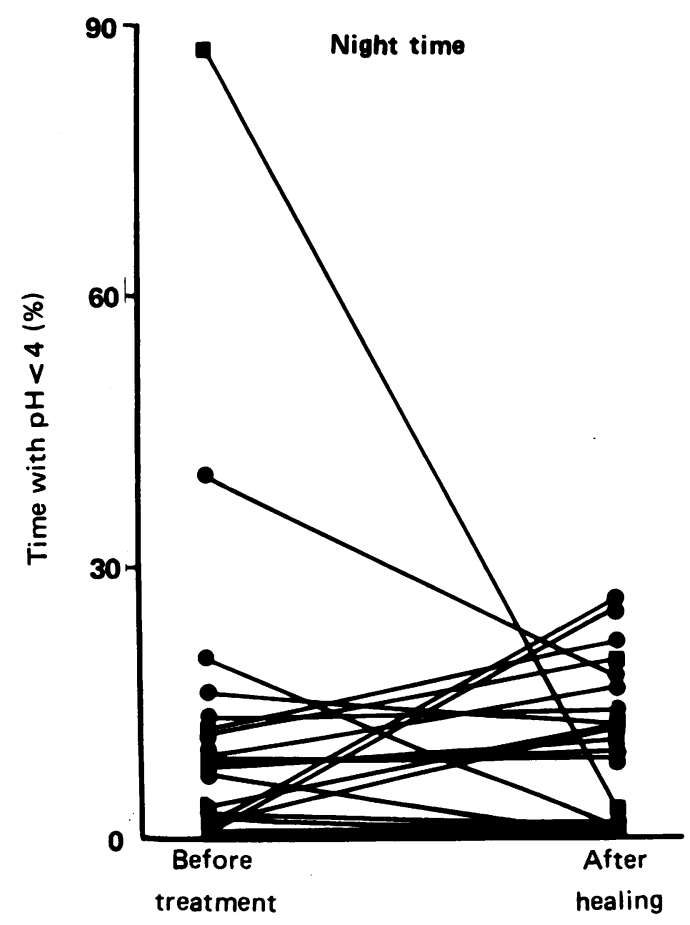

Figure 3: The percentage time with oesophageal pH<4 during night time $(8 h)$ in the 20 patients with oesophagitis before treatment and after healing of oesophagitis. For explanations of symbols, see Fig 1.

period (range 1.7-55.8). The threshold of normal for acid gastro-oesophageal reflux - that is, oesophageal $\mathrm{pH}<4$ - in our laboratory is $7 \%$ : this value yields a diagnostic sensitivity of $88.9 \%$ and a specificity of $92.9 \% .^{13}$ Only four patients had a 24 hour reflux lower than that value. Four patients showed significantly more reflux in the supine position, while the opposite was observed in 10 patients. The remaining six patients experienced a similar degree of reflux during the daytime and at night.

The median number of reflux episodes was 49.5 during the 24 hour period; corresponding values for day and for night were 38.5 and $5 \cdot 5$, respectively. Finally, the median number of reflux episodes which exceeded 5 minutes in duration during the 24 hour study was $7 \cdot 5$ (5.5 day, $2 \cdot 0$ night).

Complete improvement of symptoms was

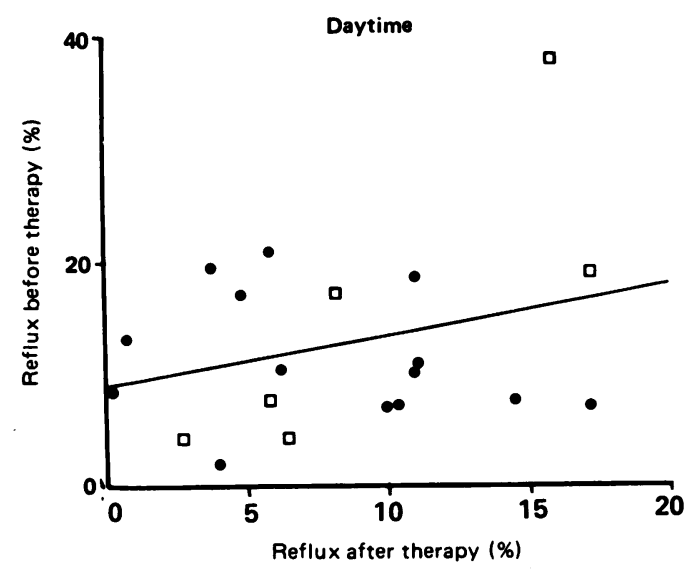

Figure 4: The relation between percentage of daytime reflux before and after treatment in the 20 patients (regression equation: $y=8 \cdot 49+0 \cdot 45 x$; significance of slope $=t=1.32$; $d f=18 ; p=0 \cdot 2)$ (see also text). For explanations of symbols, see Fig 1 .
TABLE II 24 hour results in patients before and after endoscopic healing of oesophagitis. (Data are expressed as medians)

\begin{tabular}{lrrl}
\hline & Before & After & $p$ \\
\hline Oesophageal pH<4: & & & \\
\% of total period & 10.3 & 7.8 & $>0.05$ \\
\% of daytime $(16 \mathrm{~h})$ & 10.0 & 6.5 & $<0.05$ \\
\% of night time $(8 \mathrm{~h})$ & 6.6 & 9.7 & $>0.05$ \\
Reflux episodes $(\mathrm{n}):$ & & & \\
$\quad$ During total period & 49.5 & 48.0 & $>0.05$ \\
$\quad$ During daytime & 38.5 & 38.5 & $>0.05$ \\
$\quad$ During night time & 5.5 & 8.0 & $>0.05$ \\
Reflux episodes of over 5 minutes' & & & \\
$\quad$ duration (n): & 7.5 & 4.0 & $>0.05$ \\
$\quad$ During total period & 5.5 & 1.0 & $<0.05$ \\
During daytime & 2.0 & 2.0 & $>0.05$ \\
During night time & & & \\
\hline
\end{tabular}

observed in nine patients after 12 weeks of treatment, when $12 / 20$ patients showed endoscopic healing of oesophagitis; after 24 weeks of treatment, all 20 were healed, and 14 were completely asymtomatic.

The changes in oesophageal pH parameters after healing are shown in Table II. As a group, the patients showed a non-significant reduction in median percentage time with $\mathrm{pH}<4$ during the 24 hour period $(7.8$ compared to 10.3 before treatment, $p>0.05$ ): reflux decreased in seven patients (in four of whom it returned to normal values), increased in five patients, and remained unchanged in eight (in three patients it was already within the normal range before treatment) (Fig 1). The individual data of percentage time with reflux during the daytime and night time periods are shown in Figures 2 and 3. The median daytime percentage of reflux during the day decreased significantly from $10 \%$ before treatment to $7.5 \%$ after healing $(p<0.05)$, whereas the median nocturnal reflux did not change significantly $(6.6 \% v 9.7 \%, \mathrm{p}>0.05)$.

The severity of daytime reflux tended to decrease in parallel with the pretreatment values; the trend toward a linear correlation (Fig 4; regression equation: $y=8 \cdot 49+0 \cdot 45 x)$, however, was not significant (significance of slope: $t=1 \cdot 32$; degrees of freedom $=18 ; p=0 \cdot 2$ ). As for the number of reflux episodes, no significant changes were observed in either the whole 24 hour period or in daytime and night time periods as considered separately (Table II). The number of reflux episodes of over 5 minutes' duration, however, showed after macroscopic healing similar changes to those observed in percentage time with reflux: in particular, the number of daytime episodes but not nocturnal episodes was

TABLE III 24 hour $p H$ results after acute healing in patients with and without relapse of oesophagitis. (Data are expressed as medians)

\begin{tabular}{|c|c|c|}
\hline & $\begin{array}{l}\text { Relapse } \\
(n=6)\end{array}$ & $\begin{array}{l}\text { No relapse } \\
(n=12)\end{array}$ \\
\hline \multicolumn{3}{|l|}{ Oesophageal $\mathrm{pH}<4$ : } \\
\hline$\%$ of total period & $7 \cdot 6$ & $10 \cdot 6$ \\
\hline$\%$ of daytime $(16 \mathrm{hr})$ & $7 \cdot 1$ & $9 \cdot 2$ \\
\hline$\%$ of daytime $(8 \mathrm{hr})$ & $6 \cdot 2$ & 6.9 \\
\hline \multicolumn{3}{|l|}{ Reflux episodes (n): } \\
\hline During total period & $36 \cdot 5$ & $49 \cdot 5$ \\
\hline During daytime & $33 \cdot 0$ & $38 \cdot 0$ \\
\hline During night time & $5 \cdot 5$ & 6.0 \\
\hline \multicolumn{3}{|c|}{$\begin{array}{l}\text { Reflux episodes of over } 5 \text { minutes' } \\
\text { duration }(n) \text { : }\end{array}$} \\
\hline $\begin{array}{l}\text { duration }(\mathbf{n}): \\
\text { During total period }\end{array}$ & $4 \cdot 0$ & $9 \cdot 5$ \\
\hline During daytime & $2 \cdot 5$ & $8 \cdot 0$ \\
\hline During night time & $1 \cdot 0$ & 1.5 \\
\hline
\end{tabular}


reduced compared to the pretreatment values, whereas it was not significantly changed the total 24 hour number of such reflux episodes (Table II).

To ascertain the relation between change of reflux (if any) after healing and outcome of prophylactic treatment, all the patients underwent a control endoscopy after six and 12 months of maintenance treatment with ranitidine (see Methods). Ten patients remained relapse free after one year of maintenance.treatment; six had a relapse of oesophagitis (four patients after six months, two patients after 12 months); finally, one patient dropped out and two patients continued treatment as stated by the protocol, but refused the control endoscopy because they were completely well.

The pH-metry data according to whether a relapse occurred are given in Table III. Of the six patients who relapsed, five had shown either no variation or even an increase of acid gastrooesophageal reflux at the end of the previous short term treatment, and only one patient had shown a significant reduction of reflux after healing of oesophagitis (\% time with $\mathrm{pH}<4$ during 24 hour: pretreatment $v$ posthealing value $=55 \cdot 8 \% v 11 \cdot 9 \%$ ). It must be pointed out, however, that despite the pronounced decrease, in this patient the reflux was not normal - that is, it lasted for more than $7 \%$ of total time.

\section{Discussion}

It is now commonly accepted that oesophagoscopy ( \pm biopsy) and oesophageal $\mathrm{pH}$-metry are the most accurate diagnostic tests of oesophagitis and uncomplicated gastro-oesophageal reflux, respectively. ${ }^{14}$ Often, however, inclusion criteria adopted in clinical trials with patients with gastro-oesophageal reflux disease include unreliable endoscopic signs, such as reddening, friability, granularity, and loss of glistening of the mucosa, in the absence of mucosal defects, without a positive diagnosis of pathological gastro-oesophageal reflux as defined by 24 hour pH-metry. A further problem arises in defining healing of gastro-oesophageal reflux disease, for a complete treatment success should ideally include both the disappearance of mucosal lesions and the normalisation of reflux. Severa studies $^{2-5}$ have shown, however, that medical treatment is not accompanied by an improvement in oesophageal function, even if the symptoms and the endoscopic features have been relieved, whereas surgery is followed not only by an increase in lower oesophageal sphincter pressure but also by clinical remission which persists for the entire follow up period. ${ }^{14} 15$

The consequences of these observations, if confirmed, might be of clinical importance: it could be argued, on the one hand, that surgery is the only cure for gastro-oesophageal reflux disease, but, on the other hand, that the correction of motor abnormalities is beyond the goal of medical treatment. Alternatively, it has been suggested that medical treatment may need to be continued also after endoscopic normalisation and symptomatic relief if relapse is to be avoided because gastro-oesophageal reflux is still present soon after endoscopic healing of oesophagitis.
The only two studies which included 24 hour pH-metry in the assessment of oesophageal motility changes after healing were carried out on a very limited sample of patients. We decided to undertake a study to find out in a larger series of patients whether successful treatment with the usual dose (150 mg twice daily) or higher than usual dose (300 mg twice daily) of ranitidine, administered for 12 weeks (or 24 weeks, in the case of no healing or incomplete healing), may in fact improve the pattern of gastro-oesophageal reflux compared to the pretreatment level. Furthermore, since no data on longterm outcome were provided in the above studies, we prospectively investigated the relation between relapse during longterm treatment with ranitidine and response of gastro-oesophageal reflux to short term treatment.

The results of the study indicate that reflux occurring during the daytime is significantly reduced after oesophagitis is healed. This is at variance with previous studies ${ }^{47}$ and may be explained by the higher statistical power of our study, which is due to the larger patient sample, and perhaps also by the longer duration of treatment - up to 24 weeks instead of the usual eight to 12 . This reduction in gastro-oesophageal reflux is not due to a pharmacological effect of ranitidine, since the posthealing $\mathrm{pH}$ monitoring was performed after a 'wash out' period when ranitidine was stopped for three days. Also, daytime reduction in gastro-oesophageal reflux seemed not to be related to any change in the patient's weight or smoking habit, though these were encouraged, because no significant changes in these features occurred.

Presumably, therefore, the observed reduction in gastro-oesophageal reflux is related to the macroscopic healing of the oesophageal mucosa, which in some way may have improved oesophageal motor function. This is stated by the circular theory that reflux produces injury which impairs oesophageal peristalsis and sphincter function, thus promoting more reflux. ${ }^{9}$ Our study shows that successful treatment may in fact interrupt this vicious cycle of oesophagitis, reducing the amount of reflux. This finding needs to be confirmed in a prospective study, although a similar trend has been observed in the study by Baldi et $a l,{ }^{4}$ in which the statistical power was too small to be significant.

The day to day variation of the test might hypothetically account for the apparent reduction of post-healing daytime reflux. Indeed, the concordance of two consecutive ambulatory 24 hour intraoesophageal $\mathrm{pH}$ monitoring investigations has been shown to be high, but not optimal, with a value of $83 \%$ for the daytime period and $62 \%$ for the night period, respectively. ${ }^{16}$ According to these data, we could expect a change of $\pm 1 \cdot 3 \%$ acid exposure time only as a consequence of the variability of the test (of the reflux ?). We did not test the reproducibility of $\mathrm{pH}$ monitoring, but the magnitude of the reduction we observed is almost threefold greater $(-3 \cdot 5 \%)$ than expected on the basis of the data of Johnsson and Joelsson. ${ }^{16}$

Why reflux occurring during the night is not affected by macroscopic healing is unclear. Kruse-Andersen et $a l^{17}$ have recently shown, by 
performing 12 hour simultaneous measurement of oesophageal $\mathrm{pH}$ and pressure activity in patients with oesophagitis, that apart from the three postprandial hours, the period with the highest frequency of reflux and time with acid in the oesophagus was night and that the pressure activity was also reduced. Under such circumstances, contact between the acid refluxate and the mucosa is longer if the reflux occurs when the patient is recumbent than when in the upright position, ${ }^{18}$ also because the antireflux action of gravity is absent. Thus patients with reflux that occurs mainly during the night ('supine' reflux) show a more severe oesophagitis than those with reflux that occurs mainly during the day ('upright' reflux). ${ }^{19}$

Furthermore, it has recently been observed that among the different mechanisms of gastrooesophageal reflux the absence of basal LES pressure becomes more common with increasing severity of oesophagitis, being associated with $23 \%$ of episodes in patients with complicated oesophagitis. ${ }^{20}$ In our series of patients overall the initial severity of oesophagitis was mild, with only two patients having frank ulceration of the oesophageal mucosa. This is reflected by a prevalence of only four out of 20 patients experiencing more reflux while supine.

We did not perform manometric evaluation of oesophageal peristalsis, but this has consistently been shown ${ }^{3-57}$ not to be modified by successful medical treatment, in contrast to the positive change observed after surgery. ${ }^{6}$ In our study, however, after oesophagitis healed the number of reflux episodes lasting more than 5 minutes, which is considered to reflect the clearance activity of the oesophagus after an episode of acid reflux, showed the same pattern as the percentage time with reflux - that is, a significant decrease during the day and no variation during the night compared to pretreatment values.

To date no studies using 24 hour oesophageal $\mathrm{pH}$ monitoring have been done to identify patients with reflux oesophagitis who respond or do not respond to maintenance treatment. In our study, we treated all 20 patients prophylactically with a single nocturnal dose of 150 or $300 \mathrm{mg}$ of ranitidine, for a maximum of 12 months. Interestingly, of the six patients who relapsed during maintenance treatment, five had shown either no reduction or an increase of reflux parameters during both daytime and night time after acute healing, whereas the sixth patient had had only a non-significant reduction of both frequency and duration of reflux. This finding might explain the high relapse rate reported in the studies on maintenance treatment with $\mathrm{H}_{2}$ antagonists, ${ }^{2122}$ which showed a higher figure than was observed, for example, in duodenal ulcer prophylaxis. From our data we postulate that in some patients gastro-oesophageal reflux may persist unaffected despite healing of oesophageal mucosal lesions; in such cases a relapse will occur even if patients are prophylactically treated with an $\mathrm{H}_{2}$ antagonist. In the only study on the factors that influence relapse of oesophagitis of which we are aware, ${ }^{22}$ ranitidine treatment was not found to be a relevant prognostic factor in a multiple stepwise linear regression analysis. The role of drug interactions, as well as the role of newer and more potent antisecretory agents, such as omeprazole, in the longterm treatment of reflux oesophagitis remains to be elucidated.

We conclude that our study shows that, at variance with previous data, reflux occurring during the day may be reduced by a successful course of treatment with ranitidine, provided that macroscopic healing is achieved. Patients who show such a decrease in reflux have a lower risk of relapse during maintenance treatment with the drug. Finally, the reduction in daytime gastro-oesophageal reflux seems not to be related to the severity of oesophagitis or the degree of reflux before treatment.

1 Meier RF, Sieber R, Bauerfeind P, Blum AL. Endoscopy as final arbiter in controlled clinical trials in peptic disorders. final arbiter in controlled clinical tral

2 Sonnenberg A, Lepsien G, Mueller-Lissner SA, Koelz HR, Siewert JR, Blum AL. When is esophagitis healed? Esophageal endoscopy, histology and function before and after cimetidine treatment. Dig Dis Sci 1982; 27: 297-302.

$3 \mathrm{Katz}$ PO, Knuff TE, Benjamin SB, Castell DO. Abnormal esophageal pressure in reflux oesophagitis: cause or effect? Am f Gastroenterol 1986; 81: 744-6.

4 Baldi F, Ferrarini F, Longanesi A, et al. Oesophageal function before, during, and after healing of erosive oesophagitis. Gut 1988; 29: 157-60.

5 Eckardt VF. Does healing of esophagitis improve esophageal motor function? Dig Dis Sci 1988; 33: 161-5.

6 Gill RC, Bowes KL, Murphy PD, Kingman YJ. Esophageal motor abnormalities in gastroesophageal reflux and the effects of fundoplicatio. Gastroenterology 1986; 91 : 364-9.

7 Liberman DA. 24-hour esophageal pH monitoring before and after medical therapy for reflux esophagitis. Dig Dis $S c i$ 1988; 33: 166-71

8 Eriksen CA, Sadek SA, Cranford C, Sutton D, Kennedy N, Cuschieri A. Reflux oesophagitis and oesophageal transit: evidence for a primary oesophageal motor disorder. Gut 1988; 29: 448-52.

9 Richter JE, Castell DO. Gastroesophageal reflux. Pathogenesis, diagnosis and therapy. Ann Intern Med 1982; 97: 93-103.

10 Bianchi Porro G, Pace F. Comparison of three methods of intraesophageal $\mathrm{pH}$ recordings in the diagnosis of gastroesophageal reflux. Scand $\mathcal{F}$ Gastroenterol 1988; 23: 743-50.

11 Savary M, Miller G. The esophagus. Handbook and atlas of endoscopy. Solothurn: Gassmann, 1977.

12 Emde C, Garner A, Blum AL. Technical aspects of intraluminal $\mathrm{pH}$-metry in man: current status and recommendations. Gut 1987; 28: 1177-88.

13 Schindlek NE, Neinrich C, Koenig A, Dendorfer A, Pace F, Muller-Lissner SA. Optimal thresholds, sensitivity and specificity of long-term $\mathrm{pH}$-metry for the detection of gastroesophageal reflux disease. Gastroenterology 1987; 93: $85-90$.

14 Behar J, Sheahan DG, Biancani P, Spiro HM, Storer EH. Medical and surgical management of esophagitis. A 38 month report on a prospective clinical trial. $N E$ ngl $\mathcal{Y}$ Med 1975; 293: 263-68.

15 Brand DL, Eastwood IR, Martin D, Carter WB, Pope CE. Esophageal symptoms, manometry and histology before and Esophageal symptoms, manometry and histology before and
after antireflux surgery. A long-term follow-up study. Gastroenterology 1979; 76: 1393-401.

16 Johnsson F, Joelsson B. Reproducibility of ambulatory oesophageal pH monitoring. Gut 1988; 29: 886-9.

17 Kruse-Andersen S, Wallin L, Madsen T. Acid gastrooesophageal reflux and oesophageal pressure activity during postprandial and nocturnal periods. A study in subjects with and without pathologic acid gastro-oesophageal reflux. Scand f Gastroenterol 1987; 22: 926-30.

18 DeMeester TR, Johnson LF, Joseph GJ, Toscano MS, Hall AW, Skinner DB. Patterns of gastroesophageal reflux in health and disease. Ann Surg 1976; 184: 459-70

19 DeMeester TR, Wang CI, Wernly JA, et al. Technique, indications and clinical use of 24 hour esophageal pH monitoring. F Thorac Cardiovasc Surg 1980; 79: 656-70.

20 Dent J, Holloway RH, Toouli J, Dodds WJ. Mechanisms of lower oesophageal sphincter incompetence in patients with symptomatic gastrooesophageal reflux. Gut 1988; 29: symptomati.

21 Kaul B, Petersen H, Erichsen H, et al. Gastroesophageal reflux disease. Acute and maintenance treatment with cimetidine. Scand $\mathcal{F}$ Gastroenterol 1986; 21: 139-45.

22 Koelz HR, Birchler R, Bretholz A, et al. Healing and relapse of reflux esophagitis during treatment with ranitidine. Gastroenterology 1986; 91: 1198-205. 